\title{
Application des méthodes synchrotron au domaine du patrimoine culturel
}

Marine Cotte ${ }^{(1,2)}$ (marine.cotte@esrf.fr)

(1) European Synchrotron Radiation Facility (ESRF), BP 220, 38043 Grenoble Cedex 9

(2) Laboratoire d'Archéologie Moléculaire et Structurale, CNRS UMR 8220, 94200 Ivry-sur-Seine

Les études d'objets du

patrimoine culturel par

méthodes synchrotron sont

de plus en plus fréquentes.

À I'ESRF, cette thématique a

débuté à la fin des années 90,

et est maintenant clairement

identifiée au sein de la

communauté des utilisateurs.

Cet article illustre plus

précisément les applications

de méthodes microanalytiques

(en particulier la micro-

spectroscopie d'absorption X)

dans ce domaine.

La combinaison de ces tech-

niques utilisant le rayonnement

synchrotron permet de sonder

la composition de la matière

avec une résolution submi-

cronique. Des informations

nouvelles peuvent ainsi être

obtenues, tant sur les procédés

utilisés pour la fabrication

des objets que sur les méca-

nismes d'altération qui ont pu

affecter ces objets au cours

des siècles.
À la fin des années 1990 et au début des années 2000, les études de matériaux artistiques par les méthodes utilisant le rayonnement synchrotron étaient très peu fréquentes, voire même plutôt anecdotiques. Cette thématique n'avait pas une grande visibilité, que ce soit dans la communauté synchrotron ou dans celle des physico-chimistes étudiant les œuvres d'art. Dix ans plus tard, les exemples d'analyses de peintures, verres, céramiques, métaux, manuscrits ou encore bois archéologiques par méthode synchrotron sont nombreux, et les deux communautés ont appris à parler un langage commun [1-4].

À l'ESRF, à Grenoble, une forte proportion de ces analyses est menée sur les lignes du groupe imagerie. En particulier, les lignes ID19 et ID17 accueillent régulièrement des études en paléontologie (cf. l'article de J. Baruchel et al., p. 32). Parallèlement à ces travaux, qui cherchent principalement à rendre visible l'invisible en sondant la structure tridimensionnelle des objets, d'autres expériences s'intéressent davantage à caractériser l'état chimique de la matière.

Les objets du patrimoine culturel, tels qu'ils nous apparaissent aujourd'hui, sont le résultat de nombreux procédés physiques et chimiques, depuis ceux qui ont été utilisés de manière contrôlée lors de la fabrication de l'œuvre, jusqu'à ceux qui ont modifié la matière originale par des processus de vieillissement, ou encore ceux liés aux interventions de restauration ou de conservation. C'est l'ensemble de la biographie des objets d'art (passé, présent, voire projection dans le futur) que les analyses, de laboratoire ou sur source synchrotron, vont chercher à révéler.

\section{Révéler des secrets de fabrication}

Les matériaux artistiques renferment une multitude d'informations sur la façon dont ils ont été façonnés, et l'analyse fine et précise de leurs constituants peut permettre d'identifier différents procédés de synthèse : extraction, purification, chauffage, broyage, réactions chimiques... En effet, ces procédés physico-chimiques peuvent laisser dans la matière et à différentes échelles des stigmates caractéristiques (présence d'éléments traces, état d'oxydation de certains éléments, modification des structures cristallines, produits de réaction...). Les pratiques techniques et artistiques ont évolué au cours des siècles, certaines ont même totalement disparu. L'analyse des œuvres d'art peut donc permettre d'émettre des hypothèses quant aux procédés de fabrication de ces objets. Bien souvent, des analyses semblables sont menées, en parallèle, sur des échantillons modèles, synthétisés sur la base de ces hypothèses, mais aussi de documents anciens, décrivant parfois de façon plus ou moins sibylline des recettes de fabrication. Ces matériaux modèles vont servir à identifier les stigmates et ainsi à décoder œuvres d'art et textes anciens.

À titre d'exemple, une étude a été réalisée par S. Lahlil et al. sur certains procédés d'opacification des verres [5]. Les verres analysés dans cette étude sont opaques, du fait de la présence de nanocristaux d'antimoniate de calcium. Une des hypothèses était que l'antimoine dissous dans la matrice vitreuse pouvait conserver un témoignage de la forme sous laquelle il avait été initialement introduit lors de la synthèse du verre. De nombreuses méthodes microanalytiques ont été exploitées, parmi lesquelles la micro-spectroscopie d'absorption $\mathrm{X}$, en 

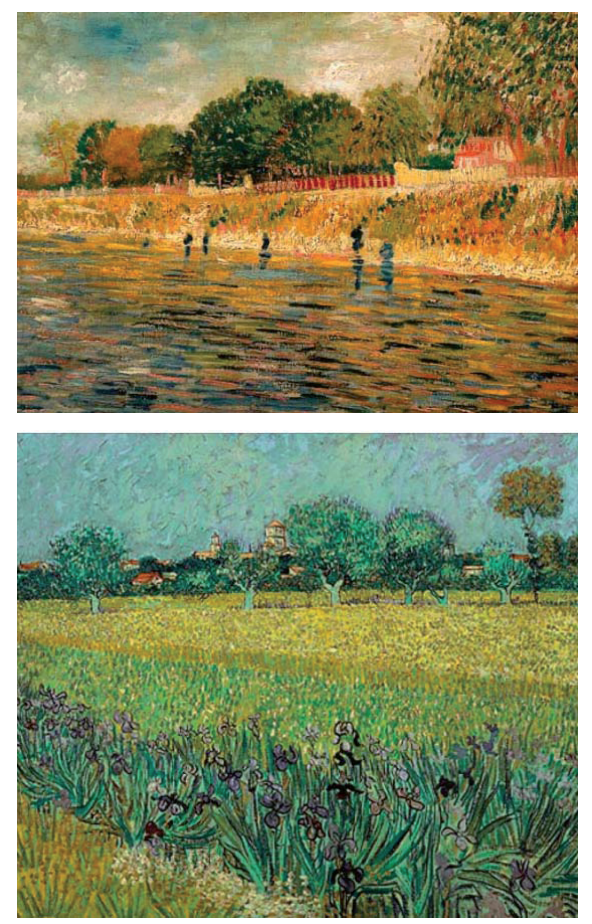

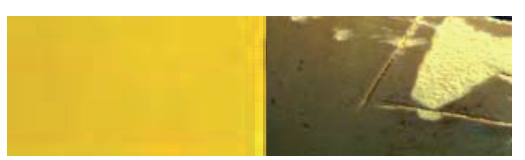

$\mathrm{PbCr}_{0,2} \mathrm{~S}_{0,8} \mathrm{O}_{4}$ orthorhombique

Fragment du $19^{\mathrm{e}}$ siècle, prélevé dans un tube

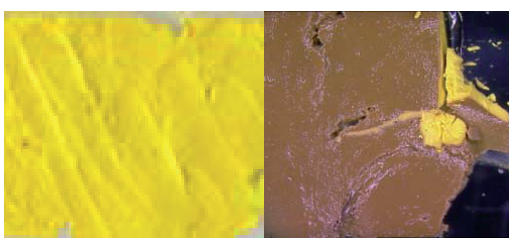

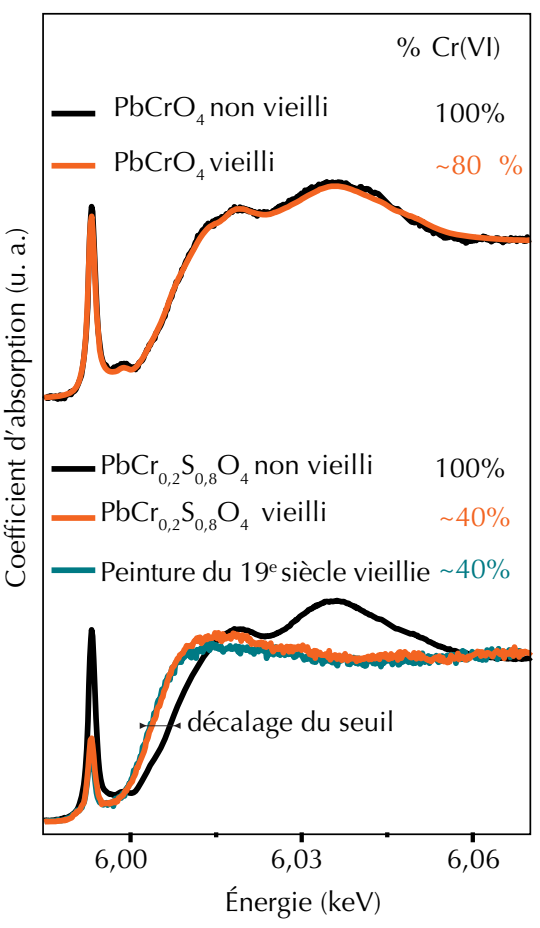

$\% \operatorname{Cr}(\mathrm{VI})$

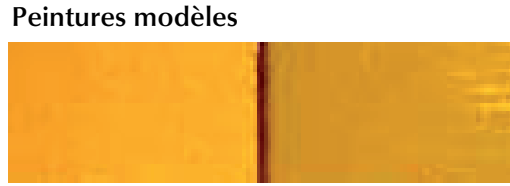

$\mathrm{PbCrO}_{4}$ monoclinique

1. Application de la microspectroscopie d'absorption X pour l'étude du brunissement du jaune de chrome dans les tableaux de Van Gogh.

À gauche, en haut : "Vue d’Arles, avec iris » (1888); en bas : « Berges de la Seine » (1887), Musée Van Gogh, Amsterdam.

Au centre : des échantillons historiques et modèles, avant et après vieillissement sous UV. II apparaît très clairement que le pigment synthétique orthorhombique et le pigment du XIXe siècle ont subi un fort brunissement en surface.

À droite : Spectres XANES (structures du coefficient d’absorption) au voisinage du seuil K du chrome, mesurés sur ces échantillons sur la ligne de lumière ID21 de I'ESRF. Le prépic à 5,993 keV est caractéristique de l'ion hexavalent $\mathrm{Cr}(\mathrm{VI})$ des chromates. La diminution de l'intensité de ce pic ainsi que le décalage vers les basses énergies de l'énergie du seuil montrent la réduction partielle du $\operatorname{Cr}(\mathrm{VI})$ en $\operatorname{Cr}(I I I)$ dans les échantillons vieillis. (D’après [6.2] et [6.4].)

particulier au voisinage d'un seuil d'absorption (XANES). Cette méthode consiste à mesurer l'absorption des rayons X par la matière en fonction de leur énergie. Elle nécessite donc de pouvoir moduler précisément $\left(\Delta \mathrm{E} / \mathrm{E} \sim 10^{-4}\right)$ et continûment (sur au moins $50 \mathrm{eV}$ ) l'énergie des rayons $\mathrm{X}$, ce qui est un des atouts majeurs des sources synchrotrons (polychromatiques continues). Des informations uniques peuvent ainsi être obtenues sur l'environnement chimique local des éléments (valence, symétrie, état d'oxydation). Dans cet exemple précis, la micro-spectroscopie d'absorption X a permis de révéler que les Égyptiens et les Romains n'utilisaient pas les mêmes procédés pour rendre les verres opaques [5]. Les premiers synthétisaient ex situ des nanocristaux d'antimoniate de calcium et les ajoutaient à la matrice vitreuse, les seconds introduisaient dans le verre une source d'antimoine, qui réagissait in situ avec le calcium pour former ces mêmes antimoniates de calcium.

Ces travaux ne se limitent pas à l'étude des matériaux artistiques. D'autres objets de la vie quotidienne, par exemple des petits flacons de produits cosmétiques, peuvent, des siècles plus tard, livrer des informations sur les pratiques et connaissances chimiques et pharmaceutiques des civilisations anciennes.

\section{Des matériaux parfois instables}

D'autres études s'intéressent à la compréhension de processus physico-chimiques responsables de la dégradation de ces œuvres, et à l'évaluation de l'efficacité de traitements de restauration ou de préservation. Ainsi, récemment, plusieurs travaux ont porté sur l'étude de l'instabilité de certains pigments utilisés, entre autres, par Van Gogh et Matisse. Au $\mathrm{XIX}^{\mathrm{e}}$ siècle, de nombreux peintres ont été attirés par les pigments synthétiques et la variété des couleurs offertes. Cependant, ils n'avaient pas alors de recul sur la stabilité à long terme de ces nouveaux composés. Plusieurs de ces pigments se sont révélés être sensibles à la lumière, qui peut modifier leur couleur (fig. 1). Dans de nombreux cas, les dégradations sont localisées principalement en surface, sous forme de couches fines de quelques microns à peine. Les analyses de microprélèvements permettent donc bien souvent de sonder non seulement les zones dégradées, mais aussi la matière originale sous-jacente, et d'identifier à la fois les ingrédients utilisés par l'artiste et d'éventuels produits ajoutés volontairement (restauration, protection...) ou non (dégradation, pollution...). Pour ce faire, la méthode la plus répandue consiste à prélever un fragment de peinture, si possible en conservant la stratigraphie complète : altération superficielle et couches originales sous-jacentes. Ce fragment, qui mesure souvent moins d'un millimètre, est enrobé dans de la résine, puis poli ou tranché de telle manière que toute la stratigraphie apparaisse en surface du bloc de résine. Pour pouvoir analyser spécifiquement chaque couche, voire grain, de ces stratigraphies complexes, deux paramètres majeurs du faisceau synchrotron sont mis à profit : la taille ultime du faisceau (quelques centaines, voire quelques dizaines de nanomètres) et son intensité ; ces deux propriétés, liées à la brillance de la source, sont indispensables pour l'acquisition de cartographies bi voire tridimensionnelles, à haute résolution.

Là encore, les analyses sur des microprélèvements faits sur les œuvres d'art peuvent être complétées par celles d'échantillons modèles préparés en 
>>

laboratoire et souvent vieillis artificiellement dans des conditions contrôlées (en jouant sur la lumière, l'humidité, la présence de polluants...). Cet aller-retour constant entre œuvre d'art et modèle permet de tester et d'affiner les hypothèses quant aux mécanismes de dégradation.

Les récents travaux de L. Monico et al. [6] sur le noircissement des chromates de plomb (pigment jaune) dans les peintures de Van Gogh sont un bon exemple de cette approche. Dans une première étape, des fragments de peinture pris dans quatre tubes datant de la fin du $\mathrm{XIX}^{\mathrm{e}}$ siècle ont été exposés aux UV. Les analyses, en particulier les mesures de l'absorption $\mathrm{X}$ au voisinage du seuil $\mathrm{K}$ du chrome, ont montré une instabilité - non systématique - du pigment, avec une réduction du chrome, de l'état hexavalent $\mathrm{Cr}(\mathrm{VI})$ à l'état trivalent $\mathrm{Cr}$ (III). Sur quatre tubes, seul celui contenant des sulfates était sensible à l'exposition aux UV [6.1]. Dans un deuxième temps, l'étude a porté sur deux fragments prélevés sur deux toiles peintes par Van Gogh : "Vue d'Arles avec iris" (1888) et «Berges de la Seine» (1887), toutes deux exposées au Musée Van Gogh d'Amsterdam (fig. 1, gauche). Les résultats étaient très similaires à ceux des échantillons vieillis artificiellement : le pigment jaune original contient des chromates, dont l'ion $\mathrm{Cr}(\mathrm{VI})$ est réduit en $\mathrm{Cr}(\mathrm{III})$ dans les zones altérées marron. De fortes teneurs en sulfates sont également observées dans ces zones altérées [6.2]. À ce stade de l'analyse, l'hypothèse était que l'altération des pigments était due à la malencontreuse association des chromates de plomb avec d'autres composés (contenant des sulfates).

Des analyses supplémentaires ont alors été réalisées, plus fines et sur un plus grand nombre de peintures de Van Gogh, qui ont révélé que ce dernier utilisait plusieurs types de pigments jaunes, ayant des teintes, des noms (jaune moyen, jaune primevère, jaune citron) et surtout des compositions différents. Certains ne contiennent que des chromates de plomb (PbCrO4) ; dans d'autres, les chromates sont partiellement remplacés par des sulfates $\left(\mathrm{PbCr}_{1-\mathrm{x}} \mathrm{S}_{\mathrm{x}} \mathrm{O}_{4}\right)$ [6.3]. Là encore, des échantillons modèles ont été synthétisés et vieillis artificiellement. On a pu prouver que ces différentes formes n'avaient pas toutes la même photosensibilité (fig. 1, milieu). La spectroscopie d'absorption au voisinage du seuil $\mathrm{K}$ du chrome a montré sans ambiguité la présence de $\sim 60 \%$ d'ions $\mathrm{Cr}$ (III) dans les couches superficielles des échantillons les plus dégradés (fig. 1, droite). L'ensemble de ces observations a permis de corriger les premières hypothèses : la dégradation des chromates de plomb dans les œuvres de Van Gogh n'est pas due à une mauvaise association de pigments (i.e. à la réaction d'un chromate de plomb avec un composé contenant des sulfates), mais plutôt au choix même de la qualité du pigment (i.e. à l'instabilité intrinsèque de certains chromates de plomb, en particulier ceux cristallisant sous forme orthorhombique) [6.4].

\section{En quoi les méthodes synchrotron sont-elles utiles ou nécessaires?}

Que ce soit pour comprendre le passé ou conserver pour le futur, une des difficultés analytiques est la taille limitée des prélèvements et leur hétérogénéité. Dans ce contexte, le faisceau synchrotron va permettre la mise en œuvre de méthodes microanalytiques classiques de laboratoire (microfluorescence X, microdiffraction des rayons $\mathrm{X}$, micro-spectroscopie infrarouge...), non destructives, avec une bien meilleure brillance, rendant possible l'acquisition de cartographies bi voire tridimensionnelles, à haute résolution.

À ces méthodes s'ajoutent toutes les techniques de spectroscopie d'absorption des rayons $\mathrm{X}$, disponibles uniquement sur source synchrotron, et qui offrent un véritable potentiel pour étudier la spéciation des éléments dans des mélanges complexes et hétérogènes [3, 4]. Sur la ligne ID21 à l'ESRF, ces techniques sont régulièrement utilisées pour analyser les matériaux artistiques et bénéficient de nombreuses innovations (comme, par exemple, un dispositif de XANES en mode plein champ, où l'ensemble de l'échantillon est éclairé par le faisceau incident).

D'une manière générale, nul doute que les développements instrumentaux en cours à l'ESRF comme dans les autres installations synchrotrons de par le monde, profiteront très bientôt à l'étude des matériaux du patrimoine.

\section{Références}

1• M. Regert, M.-F. Guerra et I. Reiche, «Physicochimie des matériaux du patrimoine culturel. Partie 2 », Techniques de l'Ingénieur, P 3781 (2006) $1-11$

2•I. Reiche, "Analyses physiques et patrimoine culturel», Bulletin de la Société Française de Physique, 153 (2006) 11-17. Téléchargeable sur le site www.refletsdelaphysique.fr

3-M. Cotte et al., "Synchrotron-Based X-ray Absorption Spectroscopy for Art Conservation: Looking Back and Looking Forward", Accounts of Chemical Research, 43 (2010) 705-714.

4 M. Cotte et J. Susini, "Art, spectres et rayons X", L'Actualité Chimique, 356-357 (2011) 113-115.

$5 \bullet$ S. Lahlil et al., "Synthesis of calcium antimonate nano-crystals by the 18th dynasty Egyptian glassmakers", Appl. Phys. A 98 (2010) 1-8.

6• L. Monico et al., "Degradation Process of Lead Chromate in Paintings by Vincent van Gogh Studied by Means of Synchrotron X-ray Spectromicroscopy and Related Methods. 1. Artificially Aged Model Samples", Analytical Chemistry 83 (2011) 12141223 ; "2. Original Paint Layer Samples", Ibid., 83 (2011) 1224-1231 ; “3. Synthesis, Characterization and Detection of Different Crystal Forms of the Chrome Yellow Pigment", Ibid., 85 (2013) 851-859; "4. Artificial Aging of Model Samples of Co-Precipitates of Lead Chromate and Lead Sulfate", Ibid., 85 (2013) 860-867.

Voir aussi le site web : www.vangogh.ua.ac.be/ 